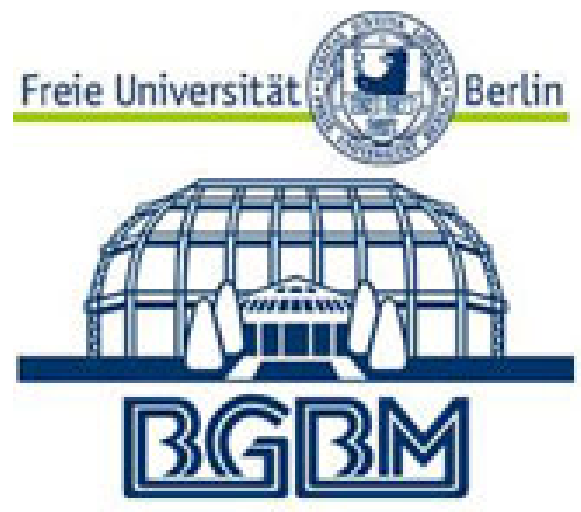

Untersuchung von Pflanzenteilen der Strychnos Dekindtiana Gilg

Author(s): H. Thoms

Source: Notizblatt des Königl. botanischen Gartens und Museums zu Berlin, Bd. 2, No. 17 (Mar. 28, 1899), pp. 260-261

Published by: Botanischer Garten und Botanisches Museum, Berlin-Dahlem

Stable URL: http://www.jstor.org/stable/3993851

Accessed: 15/06/2014 05:58

Your use of the JSTOR archive indicates your acceptance of the Terms \& Conditions of Use, available at http://www.jstor.org/page/info/about/policies/terms.jsp

JSTOR is a not-for-profit service that helps scholars, researchers, and students discover, use, and build upon a wide range of content in a trusted digital archive. We use information technology and tools to increase productivity and facilitate new forms of scholarship. For more information about JSTOR, please contact support@jstor.org. 
Der am meisten in die Augen fallende Unterschied zwischen der Strychnos cocculoides Bak. und dex Omuhahandya besteht in folgendem: bei der letzteren trägt die Rinde eine fast ebene, nur wenig rissige schwarze Korkschicht, die Frucht ist ausserordentlich bitter und enthält zahlreiche Samen, weshalb sich nur wenig Pulpa in der Frucht entwickelt flndet. Bei Strychnos cocculoides dagegen ist die Korkschicht der Rinde gelbweiss, von zahlreichen, tiefen Rissen durchzogen, die Früchte euthalten relativ nur wenige Samen und sind mit einer angenehmen, erfrischenden Pulpa erfüllt. Die Magen der Eingeborenen vertragen bis 6 dieser Früchte auf einmal ohne irgendwelche Beschwerden. Letzthin sah ich, wie ein Junge von 2 Jahren drei Mabocas (Pulpa und Samen) in wenigen Minuten ass.

Wie bei Strychnos Nux vomica L. rötet sich auch bei der Omuhahandya die Innenrinde bei Zusatz von Salpetersäure.

Das Gegengift der Eingeborenen besteht darin, dass der Kranke einige Tassen von der Abkochung der Gedärme der Ziege oder des Huhns zu sich nimmt. Diese Abkochung soll nach den eingeborenen Heilkünstlern das Gift gerade wie ein Emeto-Catharticum austreiben.

Die Eingeborenen verwenden die Omuhahandya nur bei lokalen Gliederlähmungen (Paralysis) und besonders bei schmerzenden Zuckungen des Gesichts. In diesen Fällen wird die Wurzelrinde in Pulverform zu Umschlägen verwendet, wird aber auch in kleinen Dosen (etwa eine Fingerspitze voll) innerlich genommen. -

Soweit der Bericht des Herrn Dekindt. Die mitgesandten Früchte, Samen und Rindenstiucke wurden Herrn Prof. Dr. Thoms zur chemischen Ontersuchung tibergeben, welcher das Ergebnis in folgender Mitteilung bekannt macht.

Ueber die Arten, welche Hiern ${ }^{1}$ ) als "Mabocas" auffihrt, werde ich in Bälde in Engler's Bot. Jahrb. berichten.

\section{Untersuchung von Pflanzenteilen der Strychnos Dekindtiana Gilg.}

Mitteilung aus dem Pharm.-Chemischen Laboratorium der Universität Berlin.

Von H. Thoms.

Von dem Direktor des Königl. Botanischen Museums, Herrn Geh. Regierungsrat Professor Dr. Engler, gingen mir verschiedene Pflanzen-

1) Hiorn, Wolwitsch's Plants III. p. $702 \mathrm{ff}$. 
teile der Strychnos Dekindtiana Gilg mit der Aufforderung zur Untersuchung auf Gifte zu.

Mir lagen die Frucht, sowie Wurzel und Stammrinde dieser Strychnos-Art vor. Das Material war jedoch so gering, dass an eine eingehende chemische und physiologische Prüfung auf Giftstoffe nicht gedacht werden konnte. Es musste sich vielmehr die Untersuchung auf den eventuellen Nachweis der in Strychnos-Arten vorkommenden giftigen Alkaloide, Strychnin und Brucin, beschränken. Zu dem Zwecke wurden
a. die Fruchtschale,
b. das Fruchtmus,
c. die von dem Fruchtmus durch warmes Wasser befreiten Samen,
d. die Wurzelrinde und
e. die Stammrinde

auf Alkaloide mit besonderer Rücksichtnahme auf Strychnin und Brucin geprifft.

Aus der Fruchtschale wurde in sehr kleiner Menge ein bitter schmeckender Körper isoliert, der jedoch mit Strychnin und Brucin nicht identifiziert werden konnte.

Das Fruchtmus lieferte nach der Extraktion mit Alkohol, Abdampfen des Filtrates auf dem Wasserbade und Aufnehmen mit Wasser beim Ausschütteln der alkalisch gemachten wässerigen Lösung mit Äther nach Verdampfen des letzteren einen geschmacklosen Rückstand, mit welchem Alkaloidreaktionen nicht erhalten wurden.

Hingegen liess sich in dem nicht unangenehm schmeckenden und an Tamarindenmus erinnernden Fruchtmus dieser Strychnos-Art Weinsäure in nicht unbedeutender Menge nachweisen.

Die Untersuchung der Samen auf Alkaloide lieferte ebenfalls ein negatives Resultat. (!)

Aus der Wurzel- und Stammrinde liessen sich mit Äther sehr bitter schmeckende Körper ausziehen, welche als Strychnin und Brucin aber nicht angesprochen werden konnten, da die hierfür charakteristischen Farbreaktionen nicht in voller Schärfe auftraten.

Es muss daher weiteren Untersuchungen mit grösseren Mengen Material vorbehalten bleiben, um die bitter schmeckenden Körper der Fruchtschale, der Wurzel- und Stammrinde chemisch und physiologisch eingehender prüfen zu können. 